\title{
Segregation and genetic linkage analyses of river catfish, Mystus nemurus, based on microsatellite markers
}

\author{
B.P. Hoh ${ }^{1}$, S.S. Siraj ${ }^{2}$, S.G. Tan $^{3}$ and K. Yusoff ${ }^{4,5}$ \\ ${ }^{1}$ Institute of Medical Molecular Biotechnology, Faculty of Medicine, \\ Universiti Teknologi MARA, Jalan Hospital, Sg Buloh, Selangor, Malaysia \\ ${ }^{2}$ Department of Aquaculture, Faculty of Agriculture, \\ Universiti Putra Malaysia, Serdang, Selangor, Malaysia \\ ${ }^{3}$ Department of Cell and Molecular Biology, \\ Universiti Putra Malaysia, Serdang, Selangor, Malaysia \\ ${ }^{4}$ Department of Microbiology, Universiti Putra Malaysia, Serdang, \\ Selangor, Malaysia \\ ${ }^{5}$ Institute of Biosciences, Faculty of Biotechnology and Biomolecular Sciences, \\ Universiti Putra Malaysia, Serdang, Selangor, Malaysia \\ Corresponding author: B.P. Hoh \\ E-mail: hbpeng@salam.uitm.edu.my
}

Genet. Mol. Res. 12 (3): 2578-2593 (2013)

Received April 18, 2012

Accepted November 10, 2012

Published February 28, 2012

DOI http://dx.doi.org/10.4238/2013.February.28.1

\begin{abstract}
The river catfish Mystus nemurus is an important fresh water species for aquaculture in Malaysia. We report the first genetic linkage map of $M$. nemurus based on segregation analysis and a linkage map using newly developed microsatellite markers of $M$. nemurus. A total of 70 of the newly developed polymorphic DNA microsatellite markers were analyzed on pedigrees generated using a pseudo-testcross strategy from 2 mapping families. In the first mapping family, 100 offspring were produced from randomly selected dams of the same
\end{abstract}


populations; dams of the second family were selected from 2 different populations, and this family had 50 offspring. Thirty-one of the 70 markers segregated according to the Mendelian segregation ratio. Linkage analysis revealed that 17 microsatellite markers belonging to 7 linkage groups were obtained at a logarithm of the odds score of 1.2 spanning $584 \mathrm{cM}$ by the Kosambi mapping function, whereas the other 14 remained unlinked. The results from this study will act as primer to a more extensive genetic mapping study aimed towards identifying genetic loci involved in determining economically important traits.

Key words: River catfish; Mystus nemurus; Pseudo-testcross strategy; Microsatellites; Segregation; Linkage analysis

\section{INTRODUCTION}

The river catfish Mystus nemurus is a freshwater species with great potential as an alternative fish protein source in the South East Asian region. This fish has been recognized as one of the favorite aquaculture species in Malaysia, as it is an excellent food fish with high commercial value. However, the seed supply is seasonal and its inability to reproduce in captivity is a hindrance to its mass production. State freshwater fishery hatcheries have led to research regarding induced breeding towards production of this species for distribution to fish farmers and restocking in natural water bodies throughout the country (Cheah and Thalathiah, 1993). A number of studies in the areas of genetics, nutrition and diseases have been conducted (Khan et al., 1988; Hamid et al., 2011; Kumla et al., 2012). Genetic variability and population genetic studies using starch gel electrophoresis and isozyme (Siraj et al., 1998), dominant markers such as amplified fragment length polymorphism (AFLP) and random amplified polymorphic DNA (RAPD; Chong et al., 1999), and mitochondrial DNA (Dodson et al., 1995) markers have been carried out in this species. In an attempt to generate a preliminary genetic map, we had successfully isolated and characterized DNA microsatellite markers of M. nemurus in our laboratory (Usmani et al., 2003; Chan et al., 2005; Hoh et al., 2007).

A genetic linkage map is a powerful tool for mapping quantitative trait loci of domesticated animal genomes for selective breeding purposes. It reveals mechanisms of inheritance of phenotypes that are relevant to the genetic markers. Correlations between the recombinant genome size and the physical size of the genome make it possible to estimate physical distances between markers. Comparative mapping among closely related species allows for comparison of their genomic conservation and divergence, thus providing information on genomic evolution. The most important outcome of linkage mapping is that it reveals the physical distances between markers and the distances of markers to important traits, which are useful in the isolation of genes for targeted traits.

The application of genetic markers in aquaculture research has increased dramatically in recent years. Several decades ago, protein-level genetic markers such as isozymes were commonly used in population characterization of fish and in the identification of species or hybrids (Park and Moran, 1994). Later, the discovery of various types of DNA markers, such as restriction fragment length polymorphisms (RFLP), AFLPs, RAPD, and microsatellites have 
resulted in these markers being the preferred markers used in aquaculture research.

Many aquaculture species such as tilapia (Kocher et al., 1998), rainbow trout (Young et al., 1998), kuruma prawn (Li et al., 2003), Atlantic salmon (Moen et al., 2004), walking catfish (Poompuang and Na-Nakorn, 2004), and channel catfish (Liu et al., 2003) have been mapped based on various types of molecular markers, the majority utilizing RFLP, AFLP, and RAPD. However, drawbacks exist for these markers, as they are either dominant markers and hence less informative, or time-consuming and labor intensive to use. Microsatellites, or short tandem repeats, have proven to be rather useful in constructing linkage maps. They are generally codominant and highly polymorphic, easy to score, and can be facilitated in genotyping by PCR. Microsatellite-based linkage maps have been produced for several species such as rainbow trout (Sakamoto et al., 2000) and zebrafish (Gates et al., 1999). Due to these advantages and evidence from previous studies, this marker system should produce valuable information regarding M. nemurus.

Molecular breeding and genetic mapping with the use of DNA markers have paved ways to help overcome the problems encountered in conventional breeding for improving important economic traits. Thus, an attempt was made in this study to generate the first microsatellite-based genetic linkage map of $M$. nemurus for application in selection programs through traits selected either by the use of marker-assisted selection or isolation of economically important genes.

\section{MATERIAL AND METHODS}

\section{Mapping population}

The families used in the segregation analysis of microsatellites were the crosses between 2 fishes selected randomly from the Terengganu population (hereafter referred to as family $\mathrm{A}, \mathrm{F}_{\mathrm{A}}$ ), and between a Terengganu female and a Pahang male (hereafter referred to as family $\mathrm{B}, \mathrm{F}_{\mathrm{B}}$ ). A mature female and a male parent with body weights ranging from 550 to 850 $\mathrm{g}$ were selected. Induced breeding was carried out according to the protocol of Chong et al. (1999). The females were given priming injections of the reproductive hormone, Ovaprim ${ }^{\circledR}$, twice at 8 -h intervals. The first dose was given at $0.1 \mathrm{~mL} / \mathrm{kg}$ body weight followed by the second dose at $0.4 \mathrm{~mL} / \mathrm{kg}$. The males were injected once with a dose of $0.4 \mathrm{~mL} / \mathrm{kg}$ body weight. Eight hours later, eggs and sperm were stripped from the respective males and females and mixed for dry fertilization. The eggs began hatching within $24 \mathrm{~h}$ after fertilization. Cultured Artemia nauplii were fed to the progeny twice a day for the first 2 weeks, followed by red worms (Eisenia fetida) for the following weeks. The $\mathrm{F}_{1}$ progeny were harvested at the end of the 28th day. Blood and tissue samples from the parents were also collected and DNA was extracted using the QiaAmp DNA Minikit (Qiagen, Hilden, Germany) according to manufacturer instructions.

\section{Microsatellite markers}

A total of 70 newly developed polymorphic DNA microsatellite primers (Usmani et al., 2003; Chan et al., 2005; Hoh et al., 2007, 2008) were screened in both mapping populations. The list of primers is presented in Table 1. 
Table 1. Microsatellite primer pairs and annealing temperatures (Ta) applied in the linkage analysis.

\begin{tabular}{|c|c|c|c|c|c|c|}
\hline No. & Locus name & Primers & No. of cycles & $\mathrm{Ta}\left({ }^{\circ} \mathrm{C}\right)$ & GenBank accession & References \\
\hline 1 & Mnc434a & $\begin{array}{l}\text { F: ATGGCATGCGACTAAAACA } \\
\text { R: TGGTTTTTCAGCAGTATTAG }\end{array}$ & 35 & 55 & AF346466 & Hoh, 2005 \\
\hline 2 & Mnc65b & $\begin{array}{l}\text { F: CCTGGTTTTTCAGCAGTATT } \\
\text { R: GGATCAGCATGCAACTAAA }\end{array}$ & 35 & 55 & AF346467 & Hoh, 2005 \\
\hline 3 & Mnc23 & $\begin{array}{l}\text { F: GACGGATCAGCAGCGAGGAG } \\
\text { R: ATACATCTGGTTGATGAGCA }\end{array}$ & 35 & 60 & AF478379 & Hoh, 2005 \\
\hline 4 & Mnc441 & $\begin{array}{l}\text { F: CAGGTGCAACATTTTGGAT } \\
\text { R: TTTAGAGCTATTCCCTTGGA }\end{array}$ & 35 & 55 & AF382878 & Hoh, 2005 \\
\hline 5 & Mnc340 & $\begin{array}{l}\text { F: GTCACTAGCACTGCACTTCA } \\
\text { R: TGATAAAATAAACCCGTGCT }\end{array}$ & 35 & 55 & AF412402 & Hoh, 2005 \\
\hline 6 & Mnc62 & $\begin{array}{l}\text { F: CATGGTTGTCTCAGGACAGT } \\
\text { R: GGATCAGCAAAGAATGAACA }\end{array}$ & 35 & 55 & AF346469 & Hoh, 2005 \\
\hline 7 & $\mathrm{MnVj} 2-261$ & $\begin{array}{l}\text { F: GCTGAAGGCTCCTCСТCCT } \\
\text { R: TTGAGAGCTCСТCСТCCT }\end{array}$ & 35 & 60 & AF388067 & Hoh, 2005 \\
\hline 8 & $\mathrm{MnVj2}-162$ & $\begin{array}{l}\text { F: TTTGGCAGATACGACCAC } \\
\text { R: TTCTTCCCCACTCCTCCT }\end{array}$ & 35 & 60 & AF400444 & Hoh, 2005 \\
\hline 9 & $\mathrm{MnVj2}-282$ & $\begin{array}{l}\text { F: CTGCATCAAATACAGCAACT } \\
\text { R: GAATGAGCAGCTCCTCCTC }\end{array}$ & 35 & 61 & AF402788 & Hoh, 2005 \\
\hline 10 & $\mathrm{MnVj2}-219$ & $\begin{array}{l}\text { F: GTCGAGCCTCCTCCTCCT } \\
\text { R: GACGTGCTTTCTTCACTGTC }\end{array}$ & 35 & 60 & AF402791 & Hoh, 2005 \\
\hline 11 & MnBp5-1-10b & $\begin{array}{l}\text { F: CCGGCAGAACTAGGAGTGTC } \\
\text { R: CTGTGTGAACGCTTAAAGTCAA }\end{array}$ & 35 & 55 & AY205993 & Hoh, 2005 \\
\hline 12 & MnBp5-1-05a & $\begin{array}{l}\text { F: AACACACTCTCTCTCTC } \\
\text { R: CCCTGGCTCTCCTCTACAAA }\end{array}$ & 35 & 55 & AY205990 & Hoh, 2005 \\
\hline 13 & $\mathrm{MnBp5}-1-02 \mathrm{~b}$ & $\begin{array}{l}\text { F: TCAAAGTGAGGAGATGGA } \\
\text { R: TTTTGTCACTACAGAGCTGCAT }\end{array}$ & 35 & 60 & AF526561 & Hoh, 2005 \\
\hline 14 & MnBp5-1-115b & $\begin{array}{l}\text { F: TTTTGCTACTAGAGAGACTGAC } \\
\text { R: TAGGCAAAACGTGTACTTG }\end{array}$ & 35 & 60 & AF544042 & Hoh, 2005 \\
\hline 15 & MnBp5-2-05b & $\begin{array}{l}\text { F: CAAGTGCAAAGACAGACAGA } \\
\text { R: TCTCTAAGGCTATCCATCCA }\end{array}$ & 35 & 60 & AY207448 & Hoh, 2005 \\
\hline 16 & MnBp5-2-05c & $\begin{array}{l}\text { F: TGGATGGATAGCCTTAGAGA } \\
\text { R: CCACCCAATCACTTATTTGT }\end{array}$ & 35 & 55 & AY207448 & Hoh, 2005 \\
\hline 17 & MnBp5-2-06b & $\begin{array}{l}\text { F: CGTGTCCAGACATGGTTAAT } \\
\text { R: GAGTGGGCGACTTTCAG }\end{array}$ & 35 & 55 & AY671084 & Hoh, 2005 \\
\hline 18 & MnBp5-2-22a & $\begin{array}{l}\text { F: TGTCTGAGCCAGAGAGAGA } \\
\text { R: GTCTCTGATGGTGTTTGCTT }\end{array}$ & 35 & 55 & AY205998 & Hoh, 2005 \\
\hline 19 & MnBp5-2-16a & $\begin{array}{l}\text { F: TTGCCAGCGCGAGAGA } \\
\text { R: CCTCTGTGCATTCCTCTG }\end{array}$ & 35 & 52 & AY207451 & Hoh, 2005 \\
\hline 20 & MnBp5-2-24b & $\begin{array}{l}\text { F: GTCATATTTGCTTTGGCAGT } \\
\text { R: GTGGTTTTGAATGTTCTCTG }\end{array}$ & 35 & 55 & AY207450 & Hoh, 2005 \\
\hline 21 & $\mathrm{MnBp5}-2-27 \mathrm{~b}$ & $\begin{array}{l}\text { F: TTATAACAGGGGAGTGAAGG } \\
\text { R: GATGTCATCAAGTGGCAGT }\end{array}$ & 35 & 55 & AY207449 & Hoh, 2005 \\
\hline 22 & MnBp5-3-11c & $\begin{array}{l}\text { F: CTGCATATCAAATCTGACCA } \\
\text { R: GCTGCTCGCGAGAGAGCGAGA }\end{array}$ & 35 & 55 & DQ116629 & Hoh, 2005 \\
\hline 23 & MnBp8-1-30 & $\begin{array}{l}\text { F: GGCTTATCTGTTGTTGTTG } \\
\text { R: TGAACTTTAGCCTGCTTTG }\end{array}$ & 35 & 60 & AY627196 & Hoh, 2005 \\
\hline 24 & MnBp5-1-30b & $\begin{array}{l}\text { F: TTTGGCTACTAGAGACTGACTT } \\
\text { R: GGATTATTAGGCAAAACGTG }\end{array}$ & 35 & 55 & AY852259 & Hoh, 2005 \\
\hline 25 & MnBp5-2-02a & $\begin{array}{l}\text { F: GGTCGACAGCGAGCGAGAG } \\
\text { R: TCCTGAACTGCTCAGATTTT }\end{array}$ & 35 & 55 & AF205994 & Hoh, 2005 \\
\hline 26 & MnBp5-2-02b & $\begin{array}{l}\text { F: ACACCAAAGAGATGTCCATT } \\
\text { R: TCTCTGTGAAACGCTTCTTT }\end{array}$ & 35 & 55 & AF205994 & Hoh, 2005 \\
\hline 27 & MnBp5-2-13a & $\begin{array}{l}\text { F: TCCCCGAGCGAGAGAGA } \\
\text { R: TCTGCAAGCCCTTTATAGAC }\end{array}$ & 35 & 55 & AY804209 & Hoh, 2005 \\
\hline 28 & MnBp5-2-13b & $\begin{array}{l}\text { F: CCGCTTTTTATTAGTCCTCA } \\
\text { R: CACAGAAACAGGGTTTGAA }\end{array}$ & 35 & 55 & AY804209 & Hoh, 2005 \\
\hline 29 & MnBp5-2-38b & $\begin{array}{l}\text { F: GGTTCATGCTGCTGTTTGTA } \\
\text { R: GGCTGTTACAGTAAAATACACG }\end{array}$ & 35 & 55 & AY852257 & Hoh, 2005 \\
\hline
\end{tabular}

Continued on next page 


\begin{tabular}{|c|c|c|c|c|c|c|}
\hline No. & Locus name & Primers & No. of cycles & $\mathrm{Ta}\left({ }^{\circ} \mathrm{C}\right)$ & GenBank accession & References \\
\hline 30 & MnBp8-1-10b & $\begin{array}{l}\text { F: ACCATCAGGAGGCTAAATG } \\
\text { R: GTGTTTTGTCCCCAACTTTA }\end{array}$ & 35 & 55 & AY804206 & Hoh, 2005 \\
\hline 31 & MnBp8-1-19a & $\begin{array}{l}\text { F: GCACATTTGTTGTTGTTG } \\
\text { R: CATGTAATCAAATCCCAGGT }\end{array}$ & 35 & 55 & AY804210 & Hoh, 2005 \\
\hline 32 & MnBp8-1-25a & $\begin{array}{l}\text { F: TATTCGTTGTTGTTGTTG } \\
\text { R: TGGGCTTTCAATACGTTC }\end{array}$ & 35 & 55 & AY852254 & Hoh, 2005 \\
\hline 33 & MnBp8-1-60a & $\begin{array}{l}\text { F: GTCTCTTTCGTTGTTGTT } \\
\text { R: AACCATGTGAGAAAAGGATG }\end{array}$ & 35 & 55 & AY852255 & Hoh, 2005 \\
\hline 34 & MnBp8-1-63a & $\begin{array}{l}\text { F: GGTCATCTTTGTTGTTGTTG } \\
\text { R: ACCCATCATTGGTCAGTTAG }\end{array}$ & 35 & 55 & AY852283 & Hoh, 2005 \\
\hline 35 & $\mathrm{MnVj2}-2-81$ & $\begin{array}{l}\text { F: TTAAGAGCTCTCCTCCTC } \\
\text { R: ACAGTGTAGCCCAATAAAGC }\end{array}$ & 35 & 60 & AF402788 & Hoh, 2005 \\
\hline 36 & MnBp5-2-41a & $\begin{array}{l}\text { F: TGTTTTCGAGCCAGAGAGA } \\
\text { R: GTTTCTATGAGACGTTTGTC }\end{array}$ & 35 & 55 & AY804208 & Hoh, 2005 \\
\hline 37 & MnBp5-4-20b & $\begin{array}{l}\text { F: CTTAATAGAAATGCCCGAGA } \\
\text { R: CCTATGAGACCAGCATCTTC }\end{array}$ & 35 & 55 & DQ116634 & Hoh, 2005 \\
\hline 38 & MnBp8-1-61a & $\begin{array}{l}\text { F: ACTTCGATTATTGCCTGTGT } \\
\text { R: TGCCTTTTTGTTGTTGTTG }\end{array}$ & 35 & 55 & AF860209 & Hoh, 2005 \\
\hline 39 & MnBp8-1-61b & $\begin{array}{l}\text { F: ACTTCGATTATTGCCTGTGT } \\
\text { R: TGCCTTTTTGTTGTTGTTG }\end{array}$ & 35 & 55 & AY860209 & Hoh, 2005 \\
\hline 40 & MnBp8-1-75b & $\begin{array}{l}\text { F: TTCTTCAAAGGGAAGCTAAG } \\
\text { R: CTGTTTTTGGCAGCTATCTT }\end{array}$ & 35 & 55 & AY860216 & Hoh, 2005 \\
\hline 41 & MnBp8-4-43a & $\begin{array}{l}\text { F: GTTATTTTCGTTGTTGTTG } \\
\text { R: GACCGAAGAACATAAACTAT }\end{array}$ & 35 & 55 & AY860212 & Hoh, 2005 \\
\hline 42 & MnBp8-4-43b & $\begin{array}{l}\text { F: CACTGTTGTAAGATAAATAG } \\
\text { R: GCACTGAGAAATGTGAGAAA }\end{array}$ & 35 & 55 & AY860212 & Hoh, 2005 \\
\hline 43 & MnBp8-4-43c & $\begin{array}{l}\text { F: TTTCTCACATTTCTCAGTGC } \\
\text { R: GATGTGAAGTTAACAGGTTCG }\end{array}$ & 35 & 55 & AY860212 & Hoh, 2005 \\
\hline 44 & MnBp8-4-26b & $\begin{array}{l}\text { F: GCAACTTGCACAGTATTT } \\
\text { R: ATGCGAAATTTGCACAGA }\end{array}$ & 35 & 55 & AY860214 & Hoh, 2005 \\
\hline 45 & MnBp8-4-09b & $\begin{array}{l}\text { F: CTCTCAACCTCTCCCTTTCT } \\
\text { R: TGGTCCATTTGTTGTTGTTG }\end{array}$ & 35 & 55 & AY860220 & Hoh, 2005 \\
\hline 46 & MnBp8-4-26a & $\begin{array}{l}\text { F: TTTCTGTTGTTGTTGTTG } \\
\text { R: GCACAAAATACTGTGCAA }\end{array}$ & 35 & 55 & AY860214 & Hoh, 2005 \\
\hline 47 & MnBp8-4-34a & $\begin{array}{l}\text { F: GCCTACTGTTGTTGTTGTTG } \\
\text { R: GTGGCCAGAAAAGTGTAGAA }\end{array}$ & 35 & 55 & AY806222 & Hoh, 2005 \\
\hline 48 & MnBp4-1-07a & $\begin{array}{l}\text { F: GTGGGTATCTGACACACAC } \\
\text { R: TCAGCTGACGCTGGCTATAA }\end{array}$ & 35 & 60 & AF526563 & Hoh, 2005 \\
\hline 49 & MnRm20-1 & $\begin{array}{l}\text { F: CATCACATGATCACAAGCAT } \\
\text { R: TAGTCTCCAAGTGGCTCTGT }\end{array}$ & 40 & 50 & AF462579 & Usmani, 2002 \\
\hline 50 & MnRm23-1 & $\begin{array}{l}\text { F: TTCAGACAATAGCGCTTAGA } \\
\text { R: AGTGCCCTTCATCTCTTTCT }\end{array}$ & 40 & 47 & AF462584 & Usmani, 2002 \\
\hline 51 & MnRm9-1 & $\begin{array}{l}\text { F: TGCCGTCTAACCAATCACAG } \\
\text { R: CCCCTCACAATTGTCTGTTT }\end{array}$ & 40 & 50 & AF462265 & Usmani, 2002 \\
\hline 52 & MnRmB8-1* & $\begin{array}{l}\text { F: TGTTGTGTAGAATGTGTTGTGC } \\
\text { R: CCTACCAGGGTTAGTCAGAGAGG }\end{array}$ & $3 / 3 / 33$ & $56 / 55 / 54$ & AF462242 & Usmani, 2002 \\
\hline 53 & MnRmCT6-2* & $\begin{array}{l}\text { F: TGCAGTCGACCTTAGCACAC } \\
\text { R: GCATGGACATCACATCTCTC }\end{array}$ & $3 / 3 / 33$ & $55 / 54 / 53$ & AF462266 & Usmani, 2002 \\
\hline 54 & MnRmD5-1 & $\begin{array}{l}\text { F: CATTTCTAGGCCAACATGACAG } \\
\text { R: TGTCACAGCTTCATGTATCTTCC }\end{array}$ & 40 & 50 & AF462255 & Usmani, 2002 \\
\hline 55 & MnRmE1-1* & $\begin{array}{l}\text { F: GGAGGCTGTTAGGAATTCAAAG } \\
\text { R: CGAGAGCTGAAGGCCATAAATAC }\end{array}$ & $3 / 3 / 33$ & $56 / 55 / 54$ & AF462239 & Usmani, 2002 \\
\hline 56 & MnRmE11-1* & $\begin{array}{l}\text { F: ATGTTGATGTTGTGGGATGC } \\
\text { R: GCCCAAAAAGAAACATCTGG }\end{array}$ & $3 / 3 / 33$ & $56 / 55 / 54$ & AF462241 & Usmani, 2002 \\
\hline 57 & MnRmE19-1* & $\begin{array}{l}\text { F: GGGAGGTCATTTCATTCC } \\
\text { R: TGTTTGTAGTATGGGTATGATG }\end{array}$ & $3 / 3 / 33$ & $48 / 47 / 46$ & AF462238 & Usmani, 2002 \\
\hline 58 & MnRmE2-2* & $\begin{array}{l}\text { F: CCCACGCCGTCTTTATTTC } \\
\text { R: TGTTCCGCTAGGAGGAGGAG }\end{array}$ & $3 / 3 / 33$ & $56 / 55 / 54$ & AF462554 & Usmani, 2002 \\
\hline
\end{tabular}

Continued on next page 


\begin{tabular}{|c|c|c|c|c|c|c|}
\hline No. & Locus name & Primers & No. of cycles & $\mathrm{Ta}\left({ }^{\circ} \mathrm{C}\right)$ & GenBank accession & References \\
\hline 59 & $\mathrm{MnRm} 30-1^{*}$ & $\begin{array}{l}\text { F: CTCTCAGAAGGAGTGAGCTG } \\
\text { R: GCTGTACGGAGAACAGAAAT }\end{array}$ & $3 / 3 / 33$ & $51 / 50 / 49$ & AF462586 & Usmani, 2002 \\
\hline 60 & MnRm11-1 & $\begin{array}{l}\text { F: CTTCTCCTCTGTTTCGCTGT } \\
\text { R: GGAGTGAGCTGGAGGACT }\end{array}$ & 40 & 50 & AF462582 & Usmani, 2002 \\
\hline 61 & MnRmA10-2* & $\begin{array}{l}\text { F: TCCTGCTTACCCCCTTTTTC } \\
\text { R: TGCAGAGGTGTCTCTCATCG }\end{array}$ & $3 / 3 / 30$ & $56 / 55 / 54$ & AF462264 & Usmani, 2002 \\
\hline 62 & MnSC4-1A & $\begin{array}{l}\text { F: GCCAGCAACAAGGGGCCA } \\
\text { R: CCTTGGATCGGAACTGGTC }\end{array}$ & 40 & 46 & AF458322 & Chan, 2003 \\
\hline 63 & $\mathrm{MnSC} 3-1 \mathrm{~B}$ & $\begin{array}{l}\text { F: TTCTTGCTGTGAGGCAACAG } \\
\text { R: CATAGCACGTCGTCG }\end{array}$ & 35 & 40 & AF458313 & Chan, 2003 \\
\hline 64 & Mns6-12 & $\begin{array}{l}\text { F: GATACATTAGCCATATATAA } \\
\text { R: AGTCTTTCACTTCTGCTTGA }\end{array}$ & 30 & 55 & AF325254 & Usmani, 2002 \\
\hline 65 & Mns432 & $\begin{array}{l}\text { F: AGTGCGTTAGTGTGAGTGCTTC } \\
\text { R: GAACTTTTCCCCTCCCCTTTAT }\end{array}$ & 30 & 55 & AF323792 & Usmani, 2002 \\
\hline 66 & Mns094 & $\begin{array}{l}\text { F: ATGTAATTCACTCTCCTCGCT } \\
\text { R: GATTAATACCCACAATGCACTG }\end{array}$ & 30 & 55 & AF325252 & Usmani, 2002 \\
\hline 67 & MnRh10-2 & $\begin{array}{l}\text { F: GCATTCCAGCTGAAGTGAAACA } \\
\text { R: AGGAGCCGTACAGGCTGTAATT }\end{array}$ & 30 & 55 & AF462572 & Usmani, 2002 \\
\hline 68 & $\mathrm{MnRmC} 4-2 *$ & $\begin{array}{l}\text { F: TGTCCAGTGCAGTGATGTCC } \\
\text { R: CGGGCTGTGTGTGTGTGT }\end{array}$ & $56 / 55 / 54$ & $6 / 6 / 28$ & AF462261 & Chan, 2003 \\
\hline 69 & $\operatorname{MnRm} 21-1$ & $\begin{array}{l}\text { F: GAGAGGAATCAAAGGAGAGAG } \\
\text { R: GTACTGACACGTGCACACTC }\end{array}$ & 40 & 49 & AF462587 & Usmani, 2002 \\
\hline 70 & $\mathrm{MnRm} 4-2 *$ & $\begin{array}{l}\text { F: AAAGAGTTTTATGGCAGGAGGAG } \\
\text { R: GAGAGCTGAAGGCCATAATAC }\end{array}$ & $3 / 3 / 33$ & $54 / 53 / 52$ & AF462552 & Usmani, 2002 \\
\hline
\end{tabular}

*Locus amplified by using touchdown PCR protocol.

\section{PCR amplification}

The PCR mixture consisted of $\sim 50$ ng template DNA, $10 \mathrm{mM}$ Tris- $\mathrm{HCl}, 50 \mathrm{mM} \mathrm{KCl}$, $0.1 \%$ Triton X-100, $0.2 \mathrm{mM}$ of each dNTP, 1 U Taq DNA polymerase (Promega, Madison, WI, USA), appropriate $\mathrm{MgCl}_{2}$ concentrations (ranging from 1 to $2.5 \mathrm{mM}$ ), and $\sim 10$ pmol microsatellite forward and reverse primers, and $\mathrm{ddH}_{2} \mathrm{O}$ (to a final volume of $10 \mu \mathrm{L}$ ). Amplification was performed using a PTC-200 Peltier Thermal Cycler (MJ Research, Waltham, MA, USA) and the protocol was as follows: predenaturation at $94^{\circ} \mathrm{C}$ for $3 \mathrm{~min}$, denaturation at $94^{\circ} \mathrm{C}$ for $30 \mathrm{~s}$, optimum annealing temperature for $30 \mathrm{~s}$, and extension at $72^{\circ} \mathrm{C}$ at $30 \mathrm{~s}$ for $30-40$ cycles, followed by a final extension at $72^{\circ} \mathrm{C}$ for $5 \mathrm{~min}$.

Electrophoresis was performed on 4\% Metaphor agarose (BMA, Glendale, CA, USA) with a 20-bp ladder (BMA) and permanent record was documented. For cases in which the banding patterns were unclear, $8 \%$ non-denatured polyacrylamide gel electrophoresis was applied.

\section{Analysis of inheritance and linkage studies}

Analysis of linkage between loci was performed using the JOINMAP 3.0 software package (Van Ooijen and Voorrips, 2001). Segregation of microsatellite loci was expected to be in a 1:1 ratio for markers that were heterozygous in either of the parents; ratios of 1:2:1 and 1:1:1:1 were expected for multiple alleles segregating among the parents and offspring. Map distances were calculated using Kosambi's mapping function, assuming the presence of interference, in which the interference was expected to decrease as a linear function of the distance, according to the equation 
$\mathrm{i}=1-2 \mathrm{r}$, where $i$ is the interference; $r$ is the recombination fraction. Kosambi's mapping function assumes that coincidence between crossovers is proportional to the distance between them.

\section{$\chi^{2}$ analysis of linkage}

The presence of linkage between markers was determined by summing the phenotypic data of the progeny from the 2 markers and analyzing them with the contingency chi-square test. The null hypothesis was that the segregating alleles did not deviate from Mendelian inheritance ratios under the significance level of $\mathrm{P}=0.1$. In other words, markers were considered linked if $\chi^{2}$ analyses displayed significant deviation from Mendel's law.

\section{RESULTS}

\section{Analysis of inheritance}

Before genotyping the progeny, the markers were genotyped in the parents to confirm the presence of variation (Figure 1). Of the 70 polymorphic markers tested, 31 loci displayed variation in either of the parents examined, 27 showed no variation in the parents tested, while the remaining results (12) were unscorable. The reason for the failure in amplification was not investigated further. The polymorphic markers were found to segregate according to the Mendelian inheritance pattern in the progeny. None of the progeny had alleles that were absent in the parents. Twenty-eight loci segregated according to the Mendelian inheritance ratio at a significance level of $\mathrm{P}=0.1$, while 12 deviated from Mendel's law (Table 2).

\section{$\chi^{2}$ analysis of linkage}

Linkage between the 2 loci was tested for all of the markers, and significant deviation was observed for the 13 pairs of loci (Table 3). MnBp8-4-43a, MnBp8-8-43b, and MnBp84-43c are syntenic, i.e., these loci are physically located near one another (Hoh et al., 2008). As expected, these loci exhibited significant deviations in $\chi^{2}$ tests for goodness of fit, with MnBp8-4-43a linked to MnBp8-8-43b, and MnBp8-4-43b linked to MnBp8-4-43c, although there was no significant linkage between MnBp8-4-43a and MnBp8-4-43c.

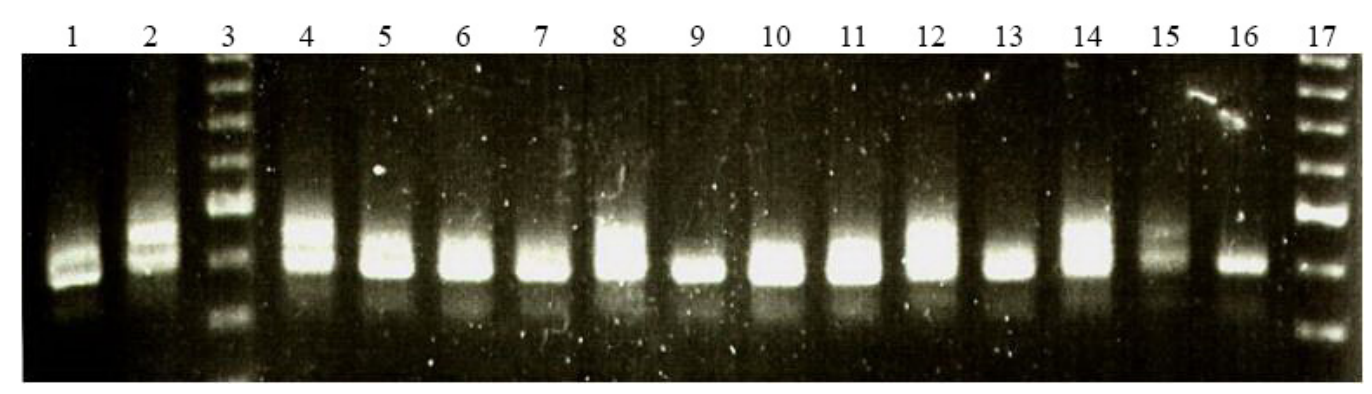

Figure 1. Genotypic profile of primer MnBp5-1-30b in family A. Lane 1 = male parent; lane 2 = female parent; lanes 4 to $16=$ progeny $\mathrm{F}_{1}$; lanes 3 and $17=20$-bp ladder. 
Table 2. Microsatellite locus genotype numbers among the $\mathrm{F}_{1}$ progeny and $\chi^{2}$ values for the expected Mendelian segregation ratio.

\begin{tabular}{|c|c|c|c|c|c|c|}
\hline Locus & $\mathrm{F}_{\mathrm{A}}$ and $\mathrm{F}_{\mathrm{B}}$ & $\begin{array}{l}\text { Parent genotype } \\
\left(\begin{array}{c}(\hat{0} \mathrm{x} \bigcirc) \\
\end{array}\right.\end{array}$ & $\begin{array}{c}\text { Expected } \\
\mathrm{F}_{1} \text { genotype ratio }\end{array}$ & $\begin{array}{c}\text { Observed } \\
\mathrm{F}_{1} \text { genotype ratio }\end{array}$ & $\chi^{2}$ & $P$ \\
\hline Mnc434a & $\mathrm{F}_{\mathrm{A}}$ & $\mathrm{BB} \times \mathrm{AB}$ & $50: 50$ & $45: 55$ & 1.000 & 0.317 \\
\hline Mnc65b & $\mathrm{F}_{\mathrm{A}}$ & $\mathrm{BB} \times \mathrm{AB}$ & $50: 50$ & $52: 48$ & 0.160 & 0.689 \\
\hline Mnc441 & $\mathrm{F}_{\mathrm{A}}$ & $\mathrm{AB} \times \mathrm{AB}$ & $25: 50: 25$ & $28: 49: 23$ & 0.540 & 0.763 \\
\hline Mnc23 & $\mathrm{F}_{\mathrm{A}}^{\mathrm{A}}$ & $\mathrm{AB} \times \mathrm{AB}$ & $25: 50: 25$ & $25: 51: 24$ & 0.060 & 0.970 \\
\hline MnBp5-1-20b & $\mathrm{F}_{\mathrm{A}}$ & $\mathrm{AC} \times \mathrm{BC}$ & $25: 25: 25: 25$ & $16: 19: 36: 29$ & 10.16 & 0.017 \\
\hline MnRm23-1 & $\mathrm{F}_{\mathrm{A}}$ & $\mathrm{AA} \times \mathrm{AB}$ & $50: 50$ & $56: 44$ & 1.44 & 0.230 \\
\hline MnRm30-1 & $\mathrm{F}_{\mathrm{B}}$ & $\mathrm{AB} \times \mathrm{AB}$ & $12.5: 25: 12.5$ & $10: 28: 12$ & 0.880 & 0.644 \\
\hline MnRm19-1 & $\mathrm{F}_{\mathrm{A}}$ & $\mathrm{AB} \times \mathrm{AB}$ & $25: 50: 25$ & $31: 40: 29$ & 4.080 & 0.130 \\
\hline MnBp5-1-5a & $\mathrm{F}_{\mathrm{A}}$ & $\mathrm{AB} \times \mathrm{AB}$ & $12.5: 25: 12.5$ & $11: 24: 15$ & 0.720 & 0.698 \\
\hline MnRm11-1 & $\mathrm{F}_{\mathrm{A}}$ & $\mathrm{AA} \times \mathrm{AB}$ & $50: 50$ & $45: 55$ & 1.000 & 0.317 \\
\hline MnBp5-2-06b & $\mathrm{F}_{\mathrm{B}}$ & $\mathrm{AA} \times \mathrm{AB}$ & $25: 25$ & $31: 19$ & 2.88 & 0.090 \\
\hline MnBp5-2-16a & $F_{B}^{B}$ & $\mathrm{AA} \times \mathrm{AB}$ & $25: 25$ & $33: 17$ & 5.120 & 0.024 \\
\hline MnRm9-1 & $F_{B}^{B}$ & $\mathrm{AA} \times \mathrm{AB}$ & $25: 25$ & $27: 23$ & 0.320 & 0.572 \\
\hline MnBp5-2-02b & $\mathrm{F}_{\mathrm{A}}^{\mathrm{B}}$ & $\mathrm{AB} \times \mathrm{AB}$ & $25: 50: 25$ & $30: 50: 20$ & 2.000 & 0.368 \\
\hline MnBp5-1-30b & $\mathrm{F}_{\mathrm{A}}$ & $\mathrm{AA} \times \mathrm{AB}$ & $50: 50$ & $68: 32$ & 12.96 & 0.000 \\
\hline MnBp8-1-60a & $\mathrm{F}_{\mathrm{A}}$ & $\mathrm{AA} \times \mathrm{AB}$ & $50: 50$ & $56: 44$ & 1.440 & 0.230 \\
\hline MnBp8-1-63a & $\mathrm{F}_{\mathrm{A}}$ & $\mathrm{AA} \times \mathrm{AB}$ & $50: 50$ & $58: 42$ & 0.256 & 0.110 \\
\hline MnBp8-1-75b & $\mathrm{F}_{\mathrm{B}}^{\mathrm{A}}$ & $\mathrm{AB} \times \mathrm{AB}$ & $10.5: 21: 10.5$ & $15: 15: 12$ & 3.857 & 0.145 \\
\hline MnBp8-4-43a & $\mathrm{F}_{\mathrm{A}}$ & $\mathrm{AA} \times \mathrm{AB}$ & $50: 50$ & $44: 56$ & 1.440 & 0.230 \\
\hline MnBp8-4-43b & $\mathrm{F}_{\mathrm{A}}$ & $\mathrm{AB} \times \mathrm{AB}$ & $25: 50: 25$ & $21: 52: 27$ & 0.880 & 0.644 \\
\hline MnBp8-4-43c & $\mathrm{F}_{\mathrm{A}}$ & $\mathrm{AA} \times \mathrm{AB}$ & $50: 50$ & $55: 45$ & 1.000 & 0.317 \\
\hline MnBp5-1-115b* & $\mathrm{F}_{\mathrm{B}}$ & $\mathrm{AB} \times \mathrm{AA}$ & $25: 25$ & $36: 14$ & 9.680 & 0.002 \\
\hline MnRmE2-2* & $\mathrm{F}_{\mathrm{B}}$ & $\mathrm{AA} \times \mathrm{AB}$ & $25: 25$ & $35: 15$ & 8.000 & 0.005 \\
\hline MnRmD5-1* & $\mathrm{F}_{\mathrm{A}}^{\mathrm{B}}$ & $\mathrm{AB} \times \mathrm{CD}$ & $25: 25: 25: 25$ & 18:19:41:20 & 14.90 & 0.002 \\
\hline MnRmE1-1* & $\mathrm{F}_{\mathrm{A}}^{\mathrm{A}}$ & $\mathrm{AB} \times \mathrm{AB}$ & $25: 50: 25$ & $11: 62: 27$ & 10.88 & 0.004 \\
\hline $\operatorname{MnRm} 20-1 *$ & $\mathrm{~F}_{\mathrm{B}}^{\mathrm{A}}$ & $\mathrm{BC} \times \mathrm{AA}$ & $25: 25$ & $12: 38$ & 13.52 & 0.000 \\
\hline MnRmE11-1* & $\mathrm{F}_{\mathrm{A}}^{\mathrm{B}}$ & $\mathrm{AB} \times \mathrm{AB}$ & $25: 50: 25$ & $33: 56: 11$ & 11.12 & 0.004 \\
\hline MnBp8-4-09b* & $\mathrm{F}_{\mathrm{A}}^{\mathrm{A}}$ & $\mathrm{AA} \times \mathrm{AB}$ & $50: 50$ & $60: 40$ & 4.000 & 0.046 \\
\hline MnBp5-4-20b* & $\mathrm{F}_{\mathrm{A}}^{\mathrm{A}}$ & $\mathrm{AA} \times \mathrm{AB}$ & $50: 50$ & $76: 24$ & 27.04 & 0.000 \\
\hline MnRmB8-1* & $\mathrm{F}_{\mathrm{B}}^{\mathrm{A}}$ & $\mathrm{AB} \times \mathrm{AB}$ & $7.5: 15: 7.5$ & $7: 9: 14$ & 8.067 & 0.018 \\
\hline $\mathrm{SC} 17 *$ & $\mathrm{~F}_{\mathrm{B}}^{\mathrm{B}}$ & $\mathrm{AA} \times \mathrm{AB}$ & $24.5: 24.5$ & $17: 32$ & 4.592 & 0.032 \\
\hline MnBp5-2-38b & $\mathrm{F}_{\mathrm{A}}$ and $\mathrm{F}_{\mathrm{B}}$ & $\mathrm{AA} \times \mathrm{AA}$ & - & - & - & - \\
\hline MnBp8-1-25a & $\mathrm{F}_{\mathrm{A}}$ and $\mathrm{F}_{\mathrm{B}}$ & $\mathrm{AA} \times \mathrm{AA}$ & - & - & - & - \\
\hline MnBp5-2-13b & $\mathrm{F}_{\mathrm{A}}$ and $\mathrm{F}_{\mathrm{B}}$ & $\mathrm{AA} \times \mathrm{AA}$ & - & - & - & - \\
\hline MnBp8-1-10b & $\mathrm{F}_{\mathrm{A}}$ and $\mathrm{F}_{\mathrm{B}}$ & $\mathrm{AA} \times \mathrm{AA}$ & - & - & - & - \\
\hline MnBp8-1-61b & $\mathrm{F}_{\mathrm{A}}$ and $\mathrm{F}_{\mathrm{B}}$ & $\mathrm{AA} \times \mathrm{AA}$ & - & - & - & - \\
\hline MnBp8-4-26b & $\mathrm{F}_{\mathrm{A}}$ and $\mathrm{F}_{\mathrm{B}}$ & $\mathrm{AA} \times \mathrm{AA}$ & - & - & - & - \\
\hline MnBp5-3-11c & $\mathrm{F}_{\mathrm{A}}$ and $\mathrm{F}_{\mathrm{B}}$ & $\mathrm{AA} \times \mathrm{AA}$ & - & - & - & - \\
\hline MnBp5-2-41a & $\mathrm{F}_{\mathrm{A}}$ and $\mathrm{F}_{\mathrm{B}}$ & $\mathrm{AA} \times \mathrm{AA}$ & - & - & - & - \\
\hline MnRmA10-2 & $\mathrm{F}_{\mathrm{A}}^{\mathrm{A}}$ and $\mathrm{F}_{\mathrm{B}}$ & $\mathrm{AA} \times \mathrm{AA}$ & - & - & - & - \\
\hline MnVj2-261 & $\mathrm{F}_{\mathrm{A}}$ and $\mathrm{F}_{\mathrm{B}}$ & $\mathrm{AA} \times \mathrm{AA}$ & - & - & - & - \\
\hline $\mathrm{MnVj2}-162$ & $\mathrm{~F}_{\mathrm{A}}$ and $\mathrm{F}_{\mathrm{B}}$ & $\mathrm{AA} \times \mathrm{AA}$ & - & - & - & - \\
\hline MnBp5-2-24b & $\mathrm{F}_{\mathrm{A}}^{\mathrm{A}}$ and $\mathrm{F}_{\mathrm{B}}$ & $\mathrm{AA} \times \mathrm{AA}$ & - & - & - & - \\
\hline MnBp5-2-27b & $\mathrm{F}_{\mathrm{A}}$ and $\mathrm{F}_{\mathrm{B}}$ & $\mathrm{AA} \times \mathrm{AA}$ & - & - & - & - \\
\hline MnVj2-219 & $\mathrm{F}_{\mathrm{A}}$ and $\mathrm{F}_{\mathrm{B}}$ & $\mathrm{AA} \times \mathrm{AA}$ & - & - & - & - \\
\hline MnBp5-2-22a & $\mathrm{F}_{\mathrm{A}}$ and $\mathrm{F}_{\mathrm{B}}$ & $\mathrm{AA} \times \mathrm{AA}$ & - & - & - & - \\
\hline MnBp5-2-02a & $\mathrm{F}_{\mathrm{A}}$ and $\mathrm{F}_{\mathrm{B}}$ & $\mathrm{AA} \times \mathrm{AA}$ & - & - & - & - \\
\hline MnBp5-1-10b & $\mathrm{F}_{\mathrm{A}}$ and $\mathrm{F}_{\mathrm{B}}$ & $\mathrm{AA} \times \mathrm{AA}$ & - & - & - & - \\
\hline MnBp8-1-30 & $\mathrm{F}_{\mathrm{A}}$ and $\mathrm{F}_{\mathrm{B}}$ & $\mathrm{AA} \times \mathrm{AA}$ & - & - & - & - \\
\hline MnBp5-2-05b & $\mathrm{F}_{\mathrm{A}}$ and $\mathrm{F}_{\mathrm{B}}$ & $\mathrm{AA} \times \mathrm{AA}$ & - & - & - & - \\
\hline MnBp5-2-05c & $\mathrm{F}_{\mathrm{A}}$ and $\mathrm{F}_{\mathrm{B}}$ & $\mathrm{AA} \times \mathrm{AA}$ & - & - & - & - \\
\hline Mnc340 & $\mathrm{F}_{\mathrm{A}}$ and $\mathrm{F}_{\mathrm{B}}$ & $\mathrm{AA} \times \mathrm{AA}$ & - & - & - & - \\
\hline Mnc62 & $\mathrm{F}_{\mathrm{A}}$ and $\mathrm{F}_{\mathrm{B}}$ & $\mathrm{AA} \times \mathrm{AA}$ & - & - & - & - \\
\hline MnRmCT6-2 & $\mathrm{F}_{\mathrm{A}}$ and $\mathrm{F}_{\mathrm{B}}$ & $\mathrm{AA} \times \mathrm{AA}$ & - & - & - & - \\
\hline $\mathrm{Sc} 12$ & $\mathrm{~F}_{\mathrm{A}}$ and $\mathrm{F}_{\mathrm{B}}$ & $\mathrm{AA} \times \mathrm{AA}$ & - & - & - & - \\
\hline SC18 & $\mathrm{F}_{\mathrm{A}}^{\mathrm{A}}$ and $\mathrm{F}_{\mathrm{B}}$ & $\mathrm{AA} \times \mathrm{AA}$ & - & - & - & - \\
\hline
\end{tabular}

Continued on next page 


\begin{tabular}{|c|c|c|c|c|c|c|}
\hline$\overline{\text { Locus }}$ & $\mathrm{F}_{\mathrm{A}}$ and $\mathrm{F}_{\mathrm{B}}$ & $\begin{array}{c}\text { Parent genotype } \\
\left(\delta \mathrm{x}^{\circ}\right)\end{array}$ & $\begin{array}{c}\text { Expected } \\
\mathrm{F}_{1} \text { genotype ratio }\end{array}$ & $\begin{array}{c}\text { Observed } \\
\mathrm{F}_{1} \text { genotype ratio }\end{array}$ & $\chi^{2}$ & $P$ \\
\hline$\overline{M n V j 2-281}$ & $\mathrm{~F}_{\mathrm{A}}$ and $\mathrm{F}_{\mathrm{B}}$ & $\mathrm{AA} \times \mathrm{AA}$ & - & - & - & - \\
\hline $\mathrm{MnVj2}-282$ & $\mathrm{~F}_{\mathrm{A}}$ and $\mathrm{F}_{\mathrm{B}}$ & $\mathrm{AA} \times \mathrm{AA}$ & - & - & - & - \\
\hline MnBp4-1-7a & $\mathrm{F}_{\mathrm{A}}$ and $\mathrm{F}_{\mathrm{B}}$ & - & - & - & - & - \\
\hline MnBp5-2-13a & $\mathrm{F}_{\mathrm{A}}$ and $\mathrm{F}_{\mathrm{B}}$ & - & - & - & - & - \\
\hline MnBp8-1-19a & $\mathrm{F}_{\mathrm{A}}$ and $\mathrm{F}_{\mathrm{B}}^{\mathrm{B}}$ & - & - & - & - & - \\
\hline MnBp8-4-34a & $\mathrm{F}_{\mathrm{A}}$ and $\mathrm{F}_{\mathrm{B}}$ & - & - & - & - & - \\
\hline $\mathrm{SC} 20^{\dagger}$ & $\mathrm{F}_{\mathrm{A}}$ and $\mathrm{F}_{\mathrm{B}}^{\mathrm{B}}$ & - & - & - & - & - \\
\hline Mns6-12 & $\mathrm{F}_{\mathrm{A}}$ and $\mathrm{F}_{\mathrm{B}}^{\mathrm{B}}$ & - & - & - & - & - \\
\hline Mns432 & $\mathrm{F}_{\mathrm{A}}$ and $\mathrm{F}_{\mathrm{B}}^{\mathrm{B}}$ & - & - & - & - & - \\
\hline Mns094 & $\mathrm{F}_{\mathrm{A}}^{\mathrm{A}}$ and $\mathrm{F}_{\mathrm{B}}^{\mathrm{B}}$ & - & - & - & - & - \\
\hline $\mathrm{MnBp} 8-4-34 \mathrm{a}^{\dagger}$ & $\mathrm{F}_{\mathrm{A}}$ and $\mathrm{F}_{\mathrm{B}}$ & - & - & - & - & - \\
\hline MnBp8-1-61b & $\mathrm{F}_{\mathrm{A}}^{\mathrm{A}}$ and $\mathrm{F}_{\mathrm{B}}^{\mathrm{B}}$ & - & - & - & - & - \\
\hline MnRh10-2 & $\mathrm{F}_{\mathrm{A}}$ and $\mathrm{F}_{\mathrm{B}}^{\mathrm{B}}$ & - & - & - & - & - \\
\hline
\end{tabular}

*Significant deviation from Medelian inheritance ratio at $\mathrm{P}=0.05$. ${ }^{\dagger}$ Unscorable/unamplified markers. $(-)=$ markers showed no amplification in the progeny. $\mathrm{F}_{\mathrm{A}}=$ Terengganu $\mathrm{x}$ Terengganu; $\mathrm{F}_{1}=100 ; \mathrm{F}_{\mathrm{B}}=$ Terengganu $\mathrm{x}$ Pahang; $\mathrm{F}_{1}=$ $50 ; \mathrm{AA}=$ homozygous genotype; $\mathrm{AB}=$ heterozygous genotype.

\begin{tabular}{|c|c|c|c|}
\hline Locus & $\chi^{2}$ & $\mathrm{P}$ & d.f. \\
\hline MnBp8-4-43a x MnBp8-4-43b & 11.742 & 0.003 & 2 \\
\hline MnBp8-4-43b x MnBp8-4-43c & 10.894 & 0.004 & 2 \\
\hline MnBp8-4-43c x MnBp5-2-02b & 18.034 & 0.000 & 2 \\
\hline MnBp8-4-43b x MnRmE19-1 & 18.300 & 0.001 & 4 \\
\hline MnBp8-1-75b x MnBp5-1-115b & 7.3210 & 0.026 & 2 \\
\hline MnBp5-1-115b x MnBp5-1-5a & 8.5400 & 0.014 & 2 \\
\hline Mnc441 x MnRm11-1 & 16.421 & 0.000 & 2 \\
\hline MnBp5-1-30b x MnBp5-4-20b & 7.1310 & 0.008 & 1 \\
\hline Mnc65b x MnBp8-1-63a & 13.800 & 0.000 & 1 \\
\hline MnRm23-1 x MnBp8-4-09b & 4.9310 & 0.026 & 1 \\
\hline MnBp8-4-09b x MnBp8-1-60a & 4.9310 & 0.026 & 1 \\
\hline MnBp5-2-16a x MnRmB30-1 & 8.7790 & 0.012 & 2 \\
\hline $\mathrm{SC} 17 \times \mathrm{MnRmB} 8-1$ & 6.1570 & 0.046 & 2 \\
\hline
\end{tabular}

Significant deviation when $\mathrm{P}<0.05$. d.f. $=$ degrees of freedom.

In addition, linkage was shown between the loci MnBp-8-4-43b and MnRmE19-1, which was physically located far apart, and segregated independently according to the Mendelian segregation. Thus, the linkage group could be outlined as shown in Figure 2.

\section{Logarithm of the odds (LOD) score analysis of linkage}

The LOD score of this study was calculated based on Kosambi's mapping function. An LOD score of significant value 3.0 and a maximum recombination fraction $(\theta) 0.499$ was set as the linkage threshold. Since we were unable to form an appropriate framework, the value was then further adjusted to a relatively less stringent criterion to form the linkage groupings. For $\mathrm{F}_{\mathrm{A}}$, a total of 12 markers were grouped at the LOD score of 1.5, forming 4 linkage groups spanning a total of $293 \mathrm{cM}$ (Kosambi's map distance; Figure 3). For $\mathrm{F}_{\mathrm{B}}$, at LOD score 1.2 (maximum recombination fraction of 0.499 ), a total of 3 linkage groups, consisting of 7 markers and spanning a total of $261 \mathrm{cM}$ (Figure 4) were obtained. Table 4 outlines the pair- 
wise analysis of the linkage between loci. The highest LOD score was found for the linkage between Mnc441 and MnRm11-1. Table 5 shows a summary of the statistics for the linkage map of the 2 M. nemurus mapping families.
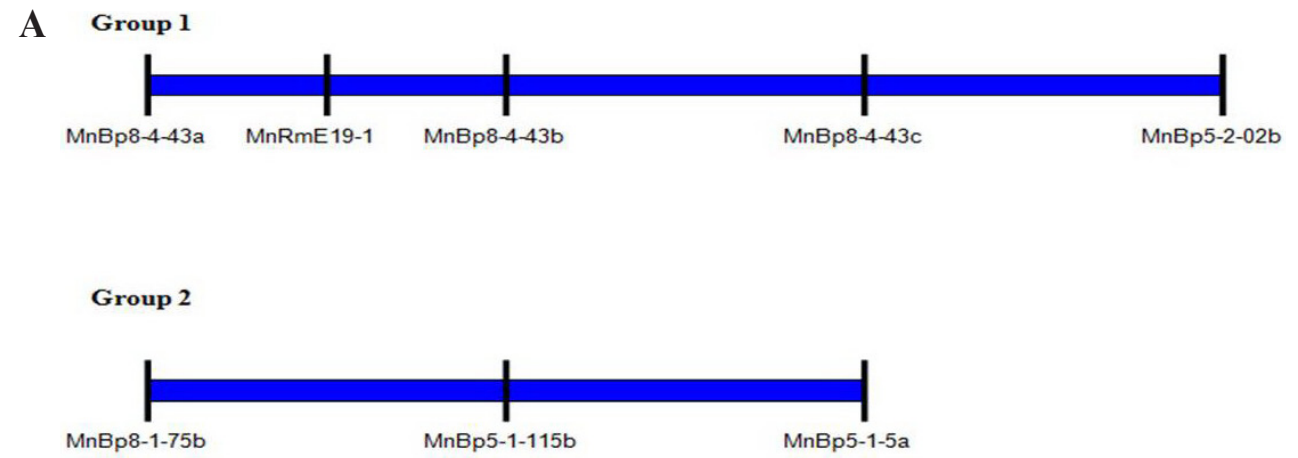

Group 3

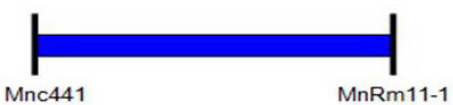

Group 4

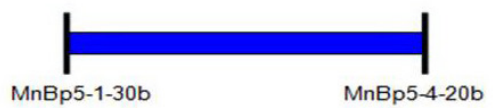

Group 5

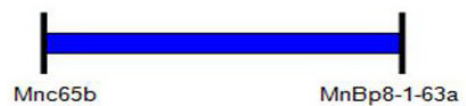

B $\quad$ Group 7
Group 6

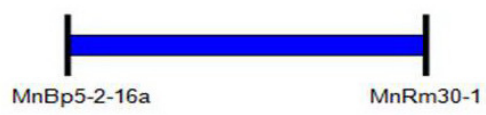

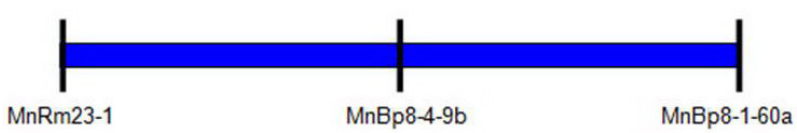

Group 8

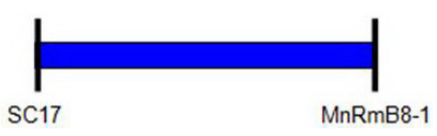

Figure 2. Linkage groups formed from the contingency chi-square analysis. 


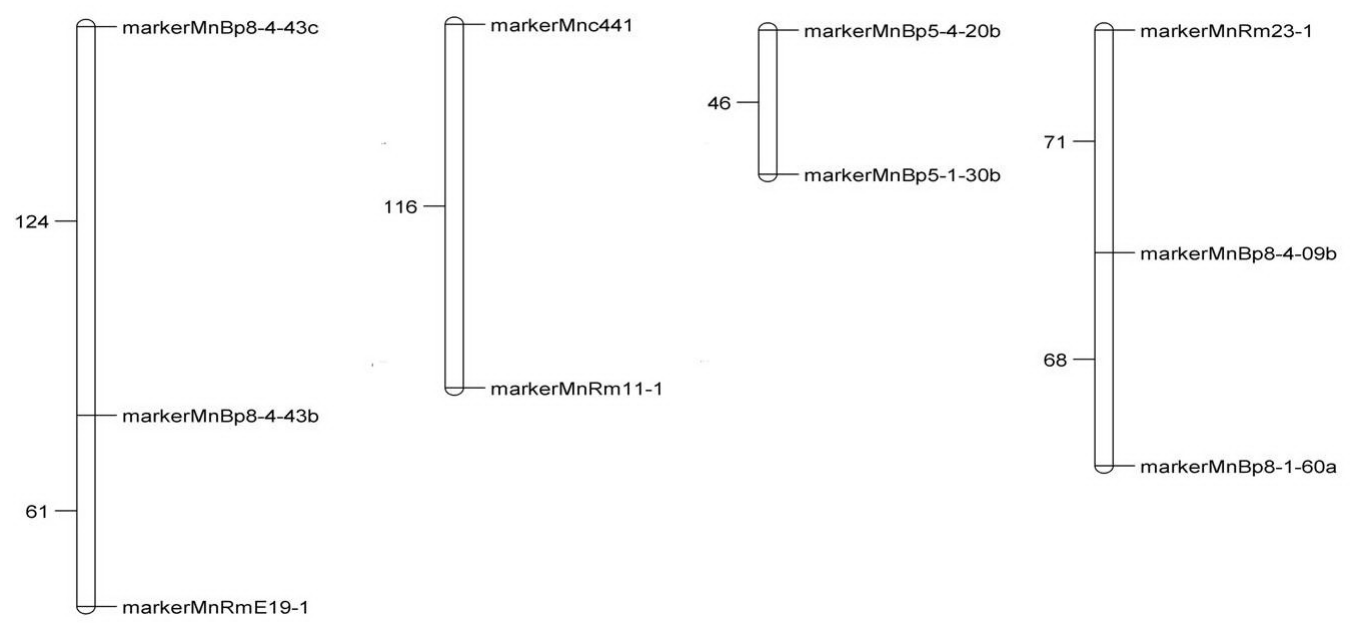

Figure 3. Linkage map generated in family A at logarithm of the odds score 1.5 using JOINMAP 3.0.

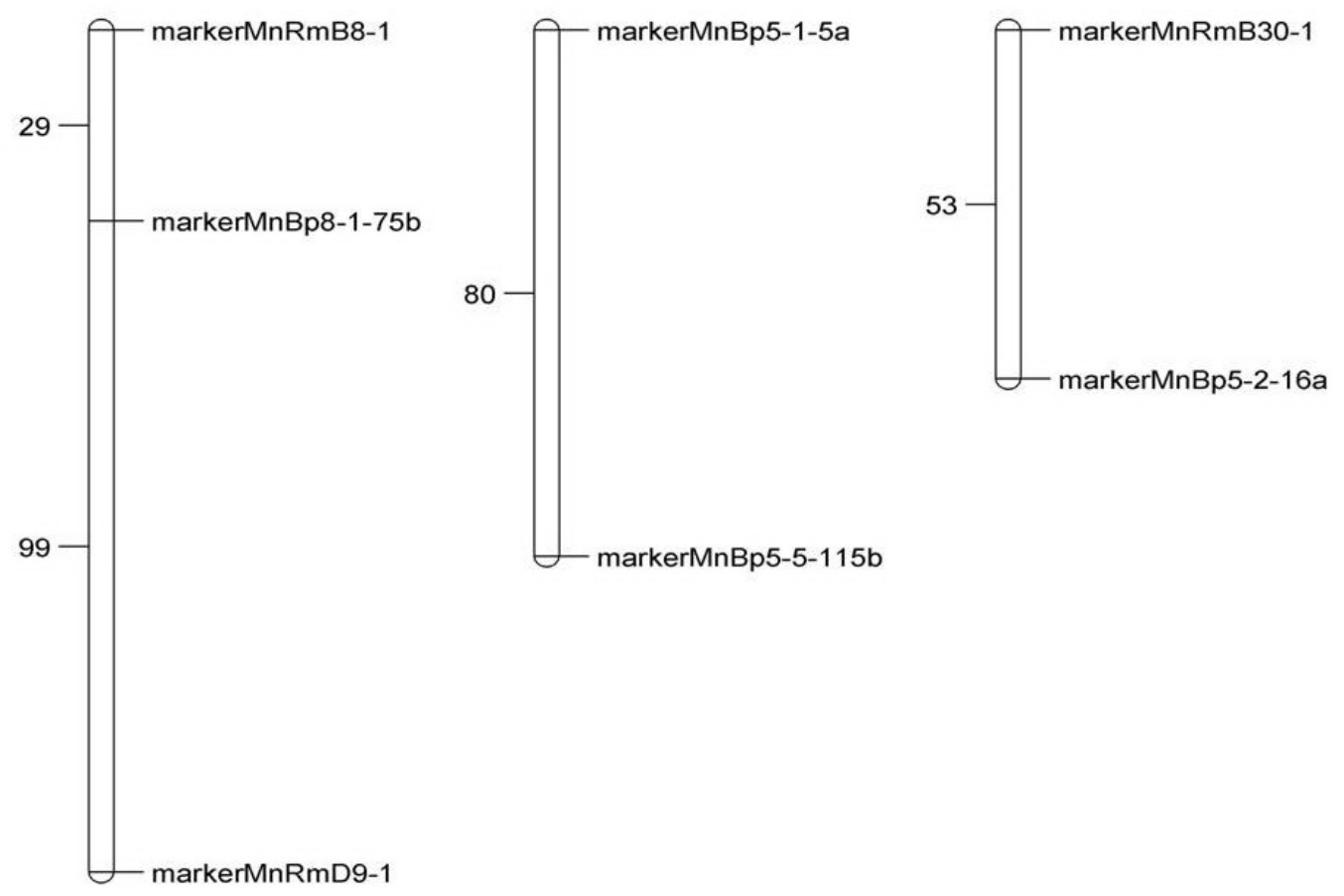

Figure 4. Linkage map generated in family B at logarithm of the odds score 1.2 using JOINMAP 3. 
Table 4. Pairwise analysis of linkage with logarithm of the odds (LOD) score and recombination fraction.

\begin{tabular}{|c|c|c|c|c|}
\hline Locus I & Locus II & $\theta$ & LOD & Group (Family) \\
\hline MnBp8-4-43b & MnRmE19-1 & 0.3512 & 1.97 & $\operatorname{Grp} 1\left(\mathrm{~F}_{\mathrm{A}}\right)$ \\
\hline MnBp8-4-43b & MnBp8-4-43c & 0.4583 & 2.28 & $\operatorname{Grp} 1\left(\mathrm{~F}_{\mathrm{A}}\right)$ \\
\hline MnRmE19-1 & MnBp8-4-43c & 0.4990 & 0.00 & Grp $1\left(F^{\prime}\right)$ \\
\hline Mnc441 & MnRm11-1 & 0.4510 & 2.64 & $\operatorname{Grp} 2\left(F^{\prime}\right)$ \\
\hline MnRm23-1 & MnRm8-4-09b & 0.3800 & 1.07 & $\operatorname{Grp} 3\left(\mathrm{~F}_{\mathrm{A}}\right)$ \\
\hline MnRm23-1 & MnBp8-1-60a & 0.4900 & 0.00 & $\operatorname{Grp} 3\left(\mathrm{~F}_{\mathrm{A}}\right)$ \\
\hline MnBp8-4-09b & MnBp8-1-60a & 0.3700 & 1.32 & $\operatorname{Grp} 3\left(F_{A}\right)$ \\
\hline MnBp5-4-20b & MnBp5-1-30b & 0.3000 & 1.48 & $\operatorname{Grp} 4\left(\mathrm{~F}_{\mathrm{A}}\right)$ \\
\hline MnRmD9-1 & MnBp8-1-75b & 0.4815 & 1.01 & Grp $1\left(\mathrm{~F}_{\mathrm{p}}\right)$ \\
\hline MnRmD9-1 & MnRmB8-1 & 0.4990 & 0.00 & $\operatorname{Grp} 1\left(\mathrm{~F}_{\mathrm{p}}\right)$ \\
\hline $\mathrm{MnBp} 8-1-75 b$ & MnRmB8-1 & 0.2630 & 1.15 & Grp $1\left(\mathrm{~F}_{\mathrm{p}}^{\prime}\right)$ \\
\hline MnBp5-1-5a & MnBp5-1-115b & 0.4615 & 1.78 & $\operatorname{Grp} 2\left(\mathrm{~F}_{\mathrm{p}}\right)$ \\
\hline MnRmB30-1 & MnBp5-2-16a & 0.3913 & 1.667 & Grp $3\left(F_{B}\right)$ \\
\hline
\end{tabular}

$\theta=$ maximum recombination fraction. For other abbreviations, see legend to Table 2.

Table 5. Summary of statistics for the linkage analysis of the mapping populations.

\begin{tabular}{lcc}
\hline & Family A & Family B \\
\hline No. of progeny & 100 & 50 \\
No. of markers & 20 & 10 \\
Linked markers & 12 & 7 \\
Unlinked markers & 8 & 3 \\
No. linkage groups & 4 & 3 \\
Markers included to map & 10 & 7 \\
Total distance & $310 \mathrm{cM}$ & $261 \mathrm{cM}$ \\
Largest group of framework & $122 \mathrm{cM}(\mathrm{Group} \mathrm{1)}$ & $128 \mathrm{cM}(\mathrm{Group} \mathrm{1)}$ \\
Smallest group of framework & $35 \mathrm{cM}(\mathrm{Group} \mathrm{4)}$ & $53 \mathrm{cM}(\mathrm{Group} \mathrm{3)}$ \\
Average distance & $77.5 \mathrm{cM}$ & $65.25 \mathrm{cM}$ \\
Logarithm of the odds score & 1.5 & 1.0 \\
\hline
\end{tabular}

\section{DISCUSSION}

In Mendelian segregation studies of microsatellite markers, segregation distortion is particularly common in aquaculture species, have been reported in several species such as kuruma prawns (Sugaya et al., 2002) and Chinook salmon (Banks et al., 1999). Possible explanations for these segregation distortions include: a) limited sample size, b) scoring errors during analysis, and c) meiotic drive, in which 2 alleles do not show Mendelian segregation from the heterozygous type (Hoh et al., 2005). Paran et al. (1995) reported a significant increase in the number of loci deviating from the expected Mendelian inheritance ratios from the $F_{2}$ to $F_{7}$ generations. They attributed this increase to a commutative effect of selection against alleles of one of the parents during the propagation of the recombinant inbred line. Gene conversion had been reported to be another cause of distortion from Mendelian expectations (Li et al., 2003). It involves the correction of heteroduplex DNA following a recombination event, which results in the copying of the sequence from that of the other homologue instead of the original sequence (Goldstein and Schlotterrer, 1999), thus causing an imbalance of segregation.

\section{Pseudo-testcross strategy}

The mapping strategy employed in this study is known as the "pseudo-testcross" strategy. 
It was first proposed by Grattapaglia and Sederoff (1994) and has been applied in numerous linkage mapping studies in plants (Grattapaglia et al., 1995; la Rosa et al., 2003). The "pseudo-testcross" refers to the fact that the testcross mating configuration of the markers is not known a priori as in a conventional testcross in which the tester is homozygous recessive for the locus of interest. Rather, the configuration is inferred to a posteriori after analyzing the parents and the genetic segregation of the marker in the progeny of a cross between 2 highly heterozygous parents when their genetic information is unavailable beforehand (Grattapaglia and Sederoff, 1994). The only requirement is sexual reproductive ability between the 2 individuals resulting in the generation of sufficient progeny to allow estimation of recombination frequencies between the segregating markers.

The major purpose of applying this strategy was to overcome the problems and the time constraint inherent in generating the $\mathrm{F}_{2}$ generation for a particular species in which the selected parents are assumed to be highly heterozygous individuals. The kuruma prawns were the first aquaculture species in which the application of this strategy was reported ( $\mathrm{Li}$ et al., 2003). However, several previous studies applied this strategy by using dominant markers (Grattapaglia and Sederoff, 1994; Kubisiak et al., 1995; Li et al., 2003). In contrast, the present study used the "pseudo-testcross" strategy to generate a linkage map using codominant single-locus DNA microsatellite markers, which were generally more useful as anchor markers than dominant markers.

This strategy is conceptually simple to implement and can be applied with any type of marker in any highly heterozygous living organism. However, the application of this strategy could be restricted by a large number of highly polymorphic population markers that are monomorphic in the parents selected to generate the mapping population. For this reason, it is desirable to perform an initial screening of marker polymorphisms on a number of broodstocks while searching for the most informative testcross configurations. Furthermore, the parents from the different populations can be crossbred as well, so as to produce a group of highly heterozygous progeny. Since this "pre-screening" approach was not applied at the earlier stage of the study, a cross between an individual from the wild Pahang population and an individual from the wild Terengganu population $\left(\mathrm{F}_{\mathrm{B}}\right)$ was carried out. This served as a "backup family" to screen for any population polymorphic marker that did not show variation in $\mathrm{F}_{\mathrm{A}}$; thus, we aimed to increase the number of markers for the linkage analysis.

\section{$\chi^{2}$ analysis of linkage}

This analysis was carried out to provide a brief review of the linkage pattern of the loci that were studied. Thirteen pairs of markers were linked to form 8 linkage groups. Several factors could have contributed to this observation. First, the physical location of the locus could have influenced the likelihood of linkage, as evidenced by the loci MnBp8-4-43a, MnBp84-43b, and MnBp8-4-43c. Secondly, alleles were likely to be associated with a phenotypic trait, and were therefore inherited together. In addition, "false linkage" should also be considered carefully. Although the "pooled" data gave no distortion, further analysis should be conducted to confirm the status of the linkage.

\section{LOD score linkage analysis}

The LOD score calculated by JoinMap for the recombination frequency is based on the $\mathrm{G}^{2}$ statistic for independence in a two-way contingency table: 


$$
\mathrm{G}^{2}=2 \Sigma \mathrm{o} \log (\mathrm{o} / \mathrm{e})
$$

where $o$ is the observed number of individuals in a cell, $e$ is the expected number of individuals in a cell, $\log$ is the natural logarithm, and $\Sigma$ is the overall sum of the cells.

Under the null hypothesis, the statistics have a chi-square distribution with the degrees of freedom (d.f.) calculated as the number of rows minus 1 , multiplied by the number of columns minus 1 . The test for independence is not affected by segregation distortion, in contrast to the LOD score typically employed in linkage analysis (i.e., the log likelihood ratio comparing the estimated value of spurious linkage). Since pairs can differ in numbers of cells in the contingency tables, the d.f. will differ as well. Therefore, $G^{2}$ statistics with d.f. $=1$ use an approximation based on equality of $\mathrm{P}$ values. Finally, the value is multiplied by 0.217 [= $\left.0.5 \mathrm{x} \log 10_{(\mathrm{e})}\right]$ to obtain the normal LOD scale. In the present study, the results presented by the contingency chi-square analysis were identical to those of the LOD analysis, supporting the accuracy of the output; thus, this finding suggested that the non-significant LOD score achieved in this study was most likely due to the insufficient number of linked markers that were genotyped. Studies with LOD score less than the significant value (3.0) had been previously reported in chickpea and potato (Bonierbale et al., 1988; Flandez-Galvez et al., 2003).

A number of the markers genotyped, although showing variability in the parental pairs, could not be assigned to the linkage map, which was most likely due to the insufficient number of markers used in the statistical calculations. Thirty-one of 70 polymorphic markers segregated according to a Mendelian ratio, of which 17 were assigned to 7 linkage groups. The river catfish genome is estimated to contain at least 25 haploid genomes (Poompuang and NaNakorn, 2004). Considering a minimum of 2 markers per linkage group, at least 50 markers are needed to generate a linkage map. Therefore, genotyping mapping populations containing more markers is essential in the near future. Numerous studies had been performed using multilocus genetic markers (AFLP, RFLP, or RAPD) to generate a linkage map (Li et al., 2003; Liu et al., 2003; la Rosa et al., 2003; Poompuang and Nanakorn, 2004). Multilocus genetic markers yield a large number of loci in a relatively short period of time, and typically with less effort, hence resulting in a genetic linkage map in a shorter amount of time. At the same time, to integrate the linkage maps constructed by the pseudo-testcross strategy, multiallelic codominant markers such as microsatellites with alleles segregating from both parents are essential as a locus bridge. Therefore, one could propose that a combination of AFLP and microsatellite markers is a more convenient and a faster approach for future genetic linkage studies in $M$. nemurus. Construction of a pure microsatellite marker linkage map could be achieved when sufficient numbers of markers are developed to replace the AFLP loci. On the other hand, the resolution of a map and the ability to determine marker order are largely dependent on population size. Clearly, population size may be technically limited by the number of DNA samples that can be reasonably prepared. Thus, a larger mapping population would provide better map resolution. Populations of less than 50 individuals generally provide too little mapping resolution to be useful (Young, 2000). For studies applying the pseudo-testcross strategy, typically 100 progeny are required to construct a significant linkage map (Grattapaglia, 1997).

The isolation of the single-locus DNA microsatellite markers used in this study was based on the 5'-anchored PCR technique (Usmani et al., 2003; Chan et al., 2005; Hoh et al., 2007, 2008). This is an interesting technique, as it detects "juxtaposed microsatellites" (Estoup et al., 1999), which are known to be syntenic. This provides an indication of the physical marker order, as syntenic markers are physically located close together, and thus usually segregate together. 
This study reports the first linkage map for M. nemurus. Nevertheless, it is far from marker saturation. Attempts are currently underway to establish a linkage map using microsatellite markers in order to analyze the correlation between the linkage groups and the chromosomes, and to identify quantitative trait loci. A genetic map is only as good as the data that were used to construct it. Depending on the quality of the data, the maps that are produced may always slightly, or even seriously, vary with the parameter settings and the selection of the subsets of loci and individuals. No mapping program can ever produce the ultimate genetic map. Whenever new data are added to existing data, the maps will change, if not with respect to order, then most likely with respect to map distance. Essentially, the calculation of a genetic linkage map is a statistical estimation procedure.

\section{ACKNOWLEDGMENTS}

Research supported by BIOTEK (grant \#01-02-04-0074) from the Ministry of Science, Technology and Innovation of Malaysia.

\section{REFERENCES}

Banks MA, Blouin MS, Baldwin BA, Rashbrook VK, et al. (1999). Isolation and inheritance of novel microsatellites in Chinook salmon (Oncorhynchus tschawytscha). J. Hered. 90: 281-288.

Bonierbale MW, Plaisted RL and Tanksley SD (1988). RFLP maps based on a common set of clones reveal modes of chromosomal evolution in potato and tomato. Genetics 120: 1095-1103.

Chan SC (2003) Development and isolation of DNA microsatellite markers for the characterization and identification of Mystus nemurus (C\&V). Master's thesis, Universiti Putra Malaysia.

Chan SC, Tan SG, Siraj SS and Yusoff K (2005). Newly developed microsatellite markers of Mystus nemurus tested for cross-species amplification in two distantly related aquacultured fish species. Asian-Australasian J. Anim. Sci. 18: 1513-1518.

Cheah SH and Thalathiah S (1993). New Technologies in Aquaculture. Proceeding of the Malaysian Fisheries Society. Occasional Publication No. 6.

Chong LK, Tan SG, Siraj SS, Christianus A, et al. (1999). Mendelian inheritance of random amplified polymorphic DNA (RAPD) markers in the river catfish (Mystus nemurus). Malays. Appl. Biol. 28: 79-84.

Dodson JJ, Colombani F and Ng PKL (1995). Phylogeographic structure in mitochondrial DNA of a South-east Asian freshwater fish, Hemibagrus nemurus (Siluroidei; Bagridae), and Pleistocene sea-level changes on the Sunda shelf. Mol. Ecol. 4: 331-346.

Estoup A, Cornuet JM, Rousset F and Guyomard R (1999). Juxtaposed microsatellite systems as diagnostic markers for admixture: theoretical aspects. Mol. Biol. Evol. 16: 898-908.

Flandez-Galvez H, Ford R, Pang EC and Taylor PW (2003). An intraspecific linkage map of the chickpea (Cicer arietinum L.) genome based on sequence tagged microsatellite site and resistance gene analog markers. Theor. Appl. Genet. 106: $1447-1456$.

Gates MA, Kim L, Egan ES, Cardozo T, et al. (1999). A genetic linkage map for zebrafish: comparative analysis and localization of genes and expressed sequences. Genome Res. 9: 334-347.

Goldstein DB and Schlotterer C (1999). Microsatellites: Evolution and Applications. Oxford University Press, New York.

Grattapaglia D (1997). Pseudo-Testcross Mapping Strategy Using RAPD Markers. In: Fingerprinting Methods Based on Arbitrarily Primed PCR (Michehli MR and Bova R, eds.). Springer-Verlag, Berlin, 201-218.

Grattapaglia D and Sederoff R (1994). Genetic linkage maps of Eucalyptus grandis and Eucalyptus urophylla using a pseudo-testcross: mapping strategy and RAPD markers. Genetics 137: 1121-1137.

Grattapaglia D, Bertolucci FL and Sederoff RR (1995). Genetic linkage mapping of QTLs controlling vegetative propagation in Eucalyptus grandis and E. urophylla using a pseudo-testcross strategy and RAPD markers. Theor. Appl. Genet. 90: 933-947.

Hamid NKA, Mahayat M and Hashim R (2011). Utilization of different carbohydrate sources and starch forms by bagrid catfish (Mystus nemurus) (Cuv \& Val). Aquac. Nutr. 17: e10-e18. 
Hoh BP (2005). Isolation, Characterization and Genetic Linkage Mapping of Microsatellite Markers Mystus nemurus. Doctoral thesis, Faculty of Science, Universiti Putra Malaysia, Selangor.

Hoh BP, Siraj SS, Tan SG and Yusoff K (2005). Mendelian inheritance of microsatellite markers in Southeast Asia river catfish, Mystus nemurus. Pertanika J. Trop. Agri. Sci. 28: 67-71.

Hoh BP, Siraj SS, Tan SG and Yusoff K (2007). Isolation and development of DNA microsatellite markers for the river catfish (Mystus nemurus). Asian Fish. Sci. 20: 41-53.

Hoh BP, Siraj SS, Tan SG and Yusoff K (2008). Isolation of trinucleotide microsatellite markers for Mystus nemurus. Genetika 44: 427-429.

Khan MS, Ambak MA and Mohsin AKM (1988). Food and feeding biology of a tropical freshwater catfish, Mystus nemurus C. \& V. with reference to its functional morphology. Indian J. Fish. 35: 78-84.

Kocher TD, Lee WJ, Sobolewska H, Penman D, et al. (1998). A genetic linkage map of a cichlid fish, the tilapia (Oreochromis niloticus). Genetics 148: 1225-1232.

Kubisiak TL, Nelson CD, Nance WL and Stine M (1995). RAPD linkage mapping in a longleaf pine x slash pine F1 family. Theo. Appl. Genet. 90: 1119-1127.

Kumla S, Doolgindachbaporn S, Sudmoon R and Sattayasai N (2012). Genetic variation, population structure and identification of yellow catfish, Mystus nemurus $(\mathrm{C} \& \mathrm{~V})$ in Thailand using RAPD, ISSR and SCAR marker. Mol. Biol. Rep. 39: 5201-5210.

la Rosa R, Angiolillo A, Guerrero C, Pellegrini M, et al. (2003). A first linkage map of olive (Olea europaea L.) cultivars using RAPD, AFLP, RFLP and SSR markers. Theor. Appl. Genet. 106: 1273-1282.

Li Y, Bayrne K, Miggiano E, Whan V, et al. (2003). Genetic mapping of the kuruma prawn Penaeus japonicus using AFLP markers. Aquaculture 219: 143-156.

Liu Z, Karsi A, Li P, Cao D, et al. (2003). An AFLP-based genetic linkage map of channel catfish (Ictalurus punctatus) constructed by using an interspecific hybrid resource family. Genetics 165: 687-694.

Moen T, Hoyheim B, Munck H and Gomez-Raya L (2004). A linkage map of Atlantic salmon (Salmo salar) reveals an uncommonly large difference in recombination rate between the sexes. Anim. Genet. 35: 81-92.

Paran I, Goldman I, Tanksley SD and Zamir D (1995). Recombinant inbred lines for genetic mapping in tomato. Theor. Appl. Genet. 90: 542-548.

Park LK and Moran P (1994). Developments in molecular genetic techniques in fisheries. Rev. Fish Biol. Fish. 4: 272-299.

Poompuang S and Na-Nakorn U (2004). A preliminary genetic map of walking catfish (Clarias macrocephalus). Aquaculture 232: 195-203.

Sakamoto T, Danzmann RG, Gharbi K, Howard P, et al. (2000). A microsatellite linkage map of rainbow trout (Oncorhynchus mykiss) characterized by large sex-specific differences in recombination rates. Genetics 155: 1331-1345.

Siraj SS, Daud SK, Othman A and Tan SG (1998). Population genetic structure of baung, Mystus nemurus (C\&V) in Malaysia. Malay. J. Appl. Biol. 27: 77-82.

Sugaya T, Ikeda M, Mori H and Taniguchi N (2002). Inheritance mode of microsatellite DNA markers and their use for kinship estimation in kuruma prawn Penaeus japonicus. Fish. Sci. 68: 299-305.

Usmani S (2002). Isolation, Characterization and Application of Microsatellite Markers in the Southeast Asian River Catfish Mystus nemurus (C\&V). Doctoral thesis, Faculty of Science, Universiti Putra Malaysia, Selangor.

Usmani S, Tan SG, Siraj SS and Yusoff K (2003). Population structure of the Southeast Asian river catfish Mystus nemurus. Anim. Genet. 34: 462-464.

Van Ooijen JW and Voorrips RE (2001). JoinMap 3.0, Software for the Calculation of Genetic Linkage Maps. Plant Research International, Wageningen.

Young ND (2000). Constructing a Plant Genetic Linkage Map with DNA Markers. In: DNA-Based Markers in Plants (Phillips RL and Vasil JK, eds.). Kluwer Academir Publishers, Alphen aan den Rijn, 31-47.

Young WP, Wheeler PA, Coryell VH, Keim P, et al. (1998). A detailed linkage map of rainbow trout produced using doubled haploids. Genetics 148: 839-850. 